\title{
Unsteady wall pressure characteristics of a 90 degree elbow in high Reynolds numbers
}

\author{
Yukiharu IWAMOTO* and Hidemasa YAMANO** \\ *Graduate School of Science and Engineering, Ehime University \\ 3 Bunkyo-cho, Matsuyama-shi, Ehime 790-8577, Japan \\ E-mail: iwamoto.yukiharu.mc@ehime-u.ac.jp \\ **Advanced Nuclear System R\&D Directorate, Japan Atomic Energy Agency \\ 4002 Narita, Oarai-shi, Ibaraki 311-1393, Japan
}

Received 29 January 2014

\begin{abstract}
Wall pressure measurements were conducted for a 90 degree elbow of which the axis curvature coincided with its inner diameter $(125 \mathrm{~mm})$. Reynolds numbers examined were $1.0 \times 10^{5}$ (available only for steady components), $3.2 \times 10^{5}$ and $5.0 \times 10^{5}$. Results showed that distributions of fluctuating normalized pressures obtained here and those made by Shiraishi et al. (2006) for the Reynolds number of $3.25 \times 10^{6}$ coincided within 0.04 of the dynamic pressure. These distributions had the same tendency: The strong fluctuating region existed in the curvature inside and had concave/convex shapes at the upstream/downstream ends, respectively. Power spectral density functions of fluctuating pressures mostly exhibited the slope of the minus seven-third law, which is seen in the inertial range of turbulence, at large frequencies. The peak spectrum with the Strouhal number of 0.5 could be found in the curvature inside downstream of the elbow. They corresponded to the vortex shedding from the boundary layer developed in the inner and aft part of the elbow. The slope at large Strouhal numbers became negatively steep near the region where the peak spectrum was seen. The peak intensity having the Strouhal number of 0.5 was quantitatively in accordance with that of the data obtained in the experimental setup that Shiraishi et al. used (Yamano et al., 2011), suggesting that the law of dynamical similarity could be applied with regard to this oscillation. Cross correlations of pressure fluctuations showed that the pressure fluctuation having the Strouhal number of 0.5 propagated as a planar wave with the bulk velocity.
\end{abstract}

Key words : 90 degree elbow, Wall pressure, Minus seven-third law, Vortex shedding, Strouhal number

\section{Introduction}

Elbows are essential piping elements and widely used in various engineering fields. Principal requirements for elbows are the flow with smaller energy loss and mechanical strength against fluid force. In order to meet the former requirement, several empirical relationships for the pressure loss yielded in an elbow (Ito, 1960, Miller, 1990 and Crawford, et al., 2007) have been proposed. Their results have shown that the optimal 90 degree elbow with minimum energy loss has its curvature of the centerline axis 2.5 times of the inner diameter. The minimum loss is achieved by a compromise between deflection and frictional losses. This configuration, however, can not be always applied because of spatial restrictions.

Fluid force acting on an elbow should be evaluated in order to satisfy the latter requirement. Steady force acting on an elbow can be predicted applying the momentum conservation theorem between far upstream and downstream flows. But unsteady force plays a more important role, especially in industrial fields, since it has possibilities to result in fatigue fractures. Unsteady fluid force acting on an elbow has been reported in such as Rütten et al. (2005) and Hirota et al. (2008). It is preferable to obtain not the force acting overall the elbow but its distribution considering effective supports. The fluid force distribution over the elbow can be obtained by wall pressure measurements.

In the present study, measurements of wall pressure distributions have been made for a 90 degree elbow of which the axis curvature coincide with its inner diameter. Reynolds numbers investigated are $1.0 \times 10^{5}$ (for steady components only), 
$3.2 \times 10^{5}$ and $5.0 \times 10^{5}$. The data of the Reynolds number of $1.0 \times 10^{5}$ are valid only for steady components because of the limitation of instrumental accuracy. Distributions of ensemble averaged (all) and root-mean-square (except $R_{e}=1.0 \times 10^{5}$ ) fluctuating pressures are given. Unsteady characteristics are mentioned in detail with power spectral density functions and cross correlation coefficients. We also compare our results with those by Shiraishi et al. (2006) which have been obtained by the elbow about 3.3 times larger than our elbow and at Reynolds numbers greater than $10^{6}$. Our results and those by Shiraishi et al. (2006) have been both obtained in a post-critical regime, where the Reynolds number is greater than the order of $10^{5}$ and a dimensionless pressure difference between an inlet and outlet of the elbow (total resistance coefficient) takes nearly constant, as reported by Idelchik (1986). Asymptotic behavior of the total resistance coefficient suggests that flow characteristics also become similar when nondimensionalized throughout the post-critical regime (whatever the Reynolds number increases). However it is little known about flow characteristics at Reynolds numbers greater than $10^{6}$. The comparisons are done in order to investigate whether the suggestion is valid at least until the Reynolds number Shiraishi et al. (2006) examined $\left(3.25 \times 10^{6}\right)$.

\section{Nomenclature}

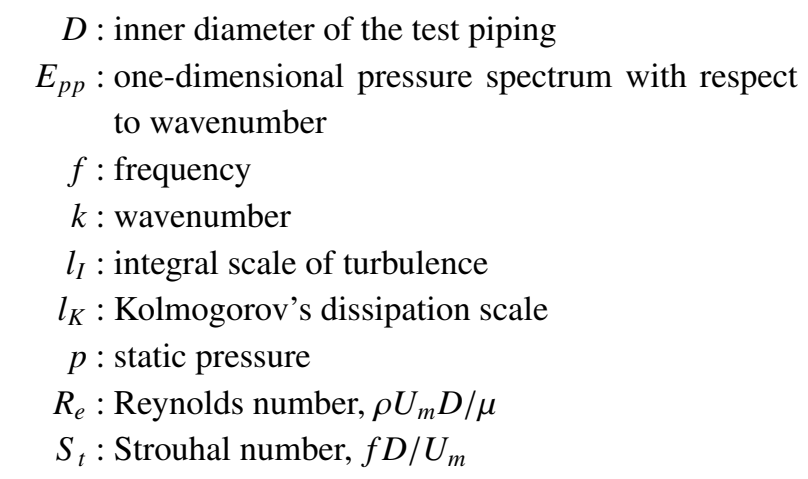

Greek letters

$\epsilon:$ turbulence dissipation rate

$\theta:$ angle from an entrance of the elbow

$\mu$ : molecular viscosity

$v:$ kinematic viscosity

$\rho$ : density

Subscripts

$B 6$ : value at Point B6 $\left(x^{\prime} / D=0, \phi=180^{\circ}\right)$

$G 1$ : value at Point $\mathrm{G} 1\left(x / D=0.5, \phi=30^{\circ}\right)$

$P S D$ : power spectral density function with respect to frequency

Superscripts

' : fluctuation component

- : ensemble average

\section{Experimental method}

The experimental apparatus is illustrated in Fig. 1. It is composed of a back pressure tank (volume: $1480 \times 980 \times$ $800 \mathrm{~mm}^{3}$, denoted by (1) in the figure), pump (Ebara 150×125FSW4H615, (2)), electro-magnetic flowmeter (Yokogawa AXF125G-NNAL1S-BJ11-0NA, AXFA11P-D101, (3)), additional buffer tank (inner diameter: 286 mm, axial length: $2000 \mathrm{~mm}$, (4)), settling chamber (5), buffer tank (inner diameter: $286 \mathrm{~mm}$, axial length: $847 \mathrm{~mm}$, (6) and test section 90 degree elbow (7). The inner diameter of the test section piping measures $125 \mathrm{~mm}$. The test section elbow has been made as a 1/10 scale model of hot leg piping of Japan sodium-cooled fast breeder reactor (JSFR), of which the conceptual design is currently carried out by the Japan Atomic Energy Agency (Kotake, et al., 2010).

Tap water with temperatures between 13 and $23{ }^{\circ} \mathrm{C}$ was used as a working fluid. Flow rates were monitored throughout the all experiments, and adjusted by an inverter (Fuji electric FRN15F1S-2J), which was connected to the pump in order to accomplish the constant Reynolds number (based on the bulk velocity and the inner diameter of the elbow). 


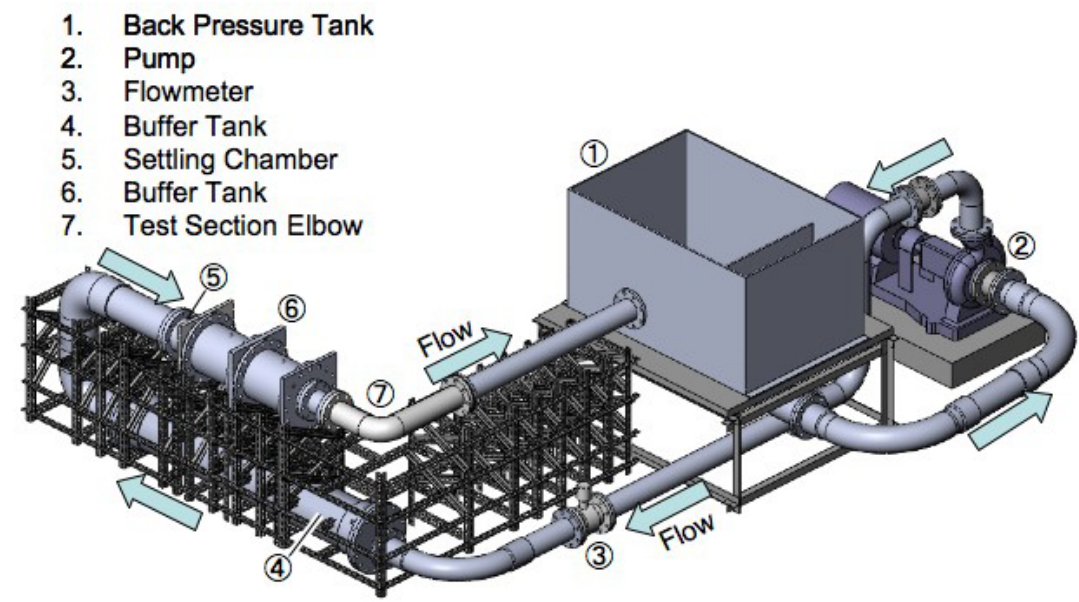

Fig. 1 Experimental apparatus

Our previous apparatus did not have the additional buffer tank (4) in Fig. 1). Pressure measurements without the additional tank showed that all the pressure signals involved periodic oscillations with $7 \pm 1$ and $12 \pm 1 \mathrm{~Hz}$. They were detected in any measuring points simultaneously and their frequencies were not affected by any flow rates and temperatures. We concluded, from these facts, that the structural vibration caused oscillations with $7 \pm 1$ and $12 \pm 1 \mathrm{~Hz}$. This kind of oscillations had been also found in experiments conducted by Ebara et al. (2010). Pressure measurements including these oscillations were not preferable, since we would like to focus only on flow induced vibrations. Therefore the additional buffer tank was installed in the present study. Preliminary experiments showed that the additional tank attenuated these oscillations, especially that with $12 \pm 1 \mathrm{~Hz}$.

Figure 2 shows pressure measurement locations in the test section elbow. Each of Cross sections $\mathrm{A}-\mathrm{O}$ has 12 pressure taps equally (30 degrees) spaced in a circumferential direction. Pressure measurements were made by connecting pressure transducers, Keyence AP-10S (repeat accuracy: $0.5 \mathrm{kPa}$ at most) and Nidec copal electronics PA-830-101G (repeat accuracy: $0.1 \mathrm{kPa}$ at most), to these pressure taps via G1/8 screws. All measurements were made after zero point adjustments that had been done in the stationary state of the fluid. These adjustments could remove effects of hydrostatic pressures that depend on altitudes of measuring points. The sampling frequency and sampling number in each pressure measurement were respectively $200 \mathrm{~Hz}$ and 21000. Almost all measuring points were measured at least twice in order to confirm repeatability of data: The data shown here were made from at least $2 \times 21000=42000$ samples.

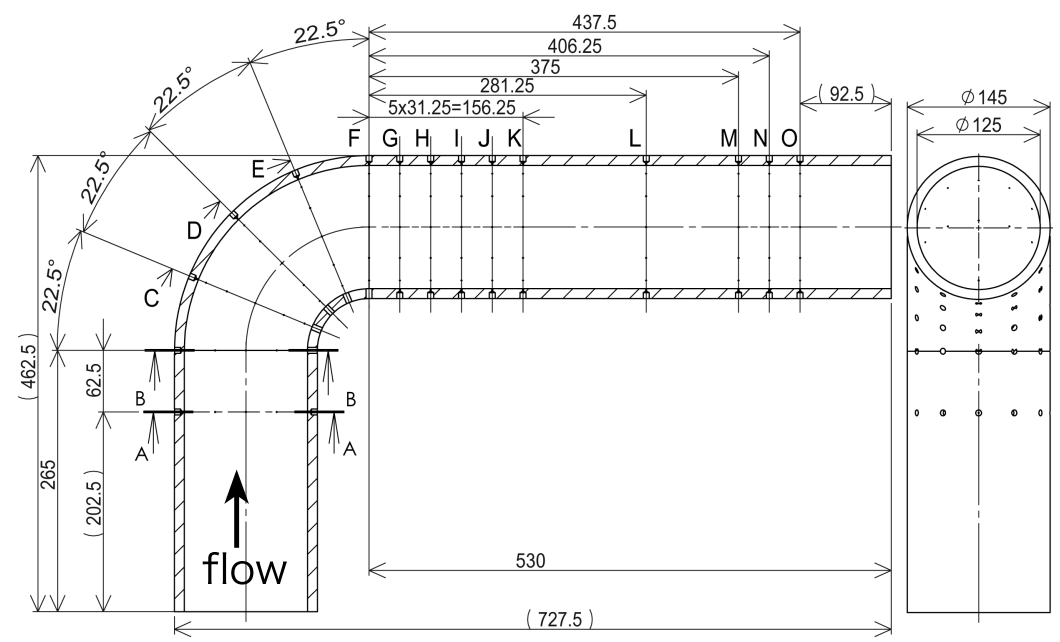

Fig. 2 Pressure measurement locations in Cross sections A - O. Every section has 12 pressure taps that are equally spaced in a circumferential direction.

We obtained power spectral density functions (PSDs) of pressure fluctuations as follows: First, obtained data were divided into five sets of them including 4096 samples. And PSDs for these five data sets were calculated by FFT analyses. Ultimate results were obtained by ensemble averaging over these PSDs. This process was effective for decreasing inci- 
dental errors. Obtained data still involved oscillations originated from the structural vibration of the experimental setup, though the aforementioned additional buffer tank weakened them. Therefore, prior to FFT analyses, we had prepared the data removing the structural vibration by subtracting reference data from raw data. The reference data could be taken at the point that was mainly affected by not the flow induced but structural vibration. This should be both far from the thickening boundary layer occurring in the aft and downstream part of the elbow and where turbulence was not be developed yet. In the present study, the outside point at the elbow inlet (Section B in Fig. 2) was selected as the reference point. Measurements were always done with that at the reference point, since the reference data should be obtained simultaneously. Ebara et al. (2010) also adopted this subtraction technique.

\section{Results and discussion}

\subsection{Pressure distributions}

Distributions of ensemble averaged and root-mean-square fluctuating pressures for Reynolds numbers $\left(R_{e}\right)$ of $1.0 \times$ $10^{5}, 3.2 \times 10^{5}$ and $5.0 \times 10^{5}(0.8,2.6$ and $4.0 \mathrm{~m} / \mathrm{s}$ in bulk velocities $)$ are respectively shown in Figs. 3 and 4 . The reference point in Fig. 3 is the outside point at the elbow inlet: The value shown in Fig. 3 is a difference between averaged pressure at every measuring point and that at the reference point. Fig. 4 has utilized not differences but raw values. Note that rootmean-square fluctuating pressures for $R_{e}=1.0 \times 10^{5}$ are not shown since instrumental errors deteriorate their quality: They have too intense scatter to be regarded as valid one. The magnitude of pressure fluctuations at $R_{e}=1.0 \times 10^{5}, 0.1$ $\mathrm{kPa}$, was smaller than accuracy of pressure transducers, within $0.5 \mathrm{kPa}$.

Ordinates in Figs. 3 and 4 show the circumferential angle $\phi$ measured from the inside of the elbow. $x^{\prime}, \theta$ and $x$ in abscissae respectively indicate the axial distance measured from the elbow inlet, angle from the elbow inlet, and distance from the elbow outlet. $D$ is the inner diameter of the elbow. Values for color contours are nondimensionalized by dynamic pressures. Small circles (o) in each figure correspond to measuring locations. The range of the ordinate is from 0 to 180 degrees, since measurements have been done in the upper half part of the elbow. Symmetry of the data has been verified by the data obtained at points 30 degrees below the center plane.

Fig. 3 illustrates that averaged pressure distributions at Reynolds numbers tried here are similar, except the elbow inside $\left(0^{\circ}<\phi<30^{\circ}, 0^{\circ}<\theta<90^{\circ}\right)$ at $R_{e}=1.0 \times 10^{5}$. The steep pressure recovery near the inside of the elbow exit $\left(\phi=0^{\circ}, \theta=67.5^{\circ}\right.$ ), making contour boundaries from blue to green be concave to the downstream region, can be seen in Figs. 3(b) and (c). On the other hand gradual pressure recovery, making contour boundaries from blue to green be concentric circles, is seen in Fig. 3(a). The difference probably is caused by whether the flow separation occurs or not. Our previous LDV velocity measurements under the case of $R_{e}=3.2 \times 10^{5}$ has shown the flow separation in the downstream region of the elbow (Iwamoto, et al., 2012). Fig. 4 illustrates that root-mean-square values for $R_{e}=3.2 \times 10^{5}$ and $5.0 \times 10^{5}$ also have similar distributions.

As mentioned above, the test section elbow has been made as a 1/10 scale model of hot leg piping of JSFR. Another test using a $1 / 3$ scale model has been done by Shiraishi et al. (2006). They clarified the distribution of root-mean-square pressure fluctuations at $R_{e}=3.25 \times 10^{6}(9.28 \mathrm{~m} / \mathrm{s}$ in the bulk velocity). It is adapted here in Fig. 5 . It should be noted that following modifications have been done by authors in order to make comparison easy and accommodate our definition: (1) coloring of contours, (2) recalculation of values in the ordinate and abscissa, and (3) change of labeling of Cross sections $\mathrm{A}-\mathrm{O}$, which are shown in Fig. 2. We also emphasize that increments in the abscissa in Fig. 5 are uneven.

Comparison between Figs. 4 and 5 indicates that they coincide within 0.04 of the dynamic pressure. Especially, they have common characteristics that values become maximum near points $0.5 \mathrm{D}$ downstream and inside elbows and that contours surrounding maximum values are convex at left sides. These characteristics are caused by vortex shedding from the separated boundary layer. Figs. 4 and 5 show dynamically similar flows in experimental apparatuses with different sizes and bulk velocities. The coincidence between Figs. 4 and 5 also guarantees accuracies of measurements in Fig. 4. Raw data in Fig. 4 had magnitude of $0.1 \mathrm{kPa}$. So we can conclude that our pressure transducers utilized are able to capture the fluctuation greater than $0.1 \mathrm{kPa}$, whereas their accuracy is nominally within $0.5 \mathrm{kPa}$.

\subsection{Power spectral density functions of pressure fluctuations}

Figure 6 shows power spectral density functions (PSDs) of pressure fluctuations, $p_{P S D}^{\prime}$, at inside and outside locations in cases of $R_{e}=3.2 \times 10^{5}$ and $5.0 \times 10^{5}$. Letters A0 - M6 written in each figure in Fig. 6 indicate measuring points: The alphabet means cross section in Fig. 2 and numbers are circumferentially assigned from the intrados of the elbow. Results except Point B6 (the entrance and outside of the elbow, shown in the upper right in Fig. 6 (b)) have been generated from data reducing structural vibrations. These were made by subtracting the reference data, i.e. simultaneously measured data 


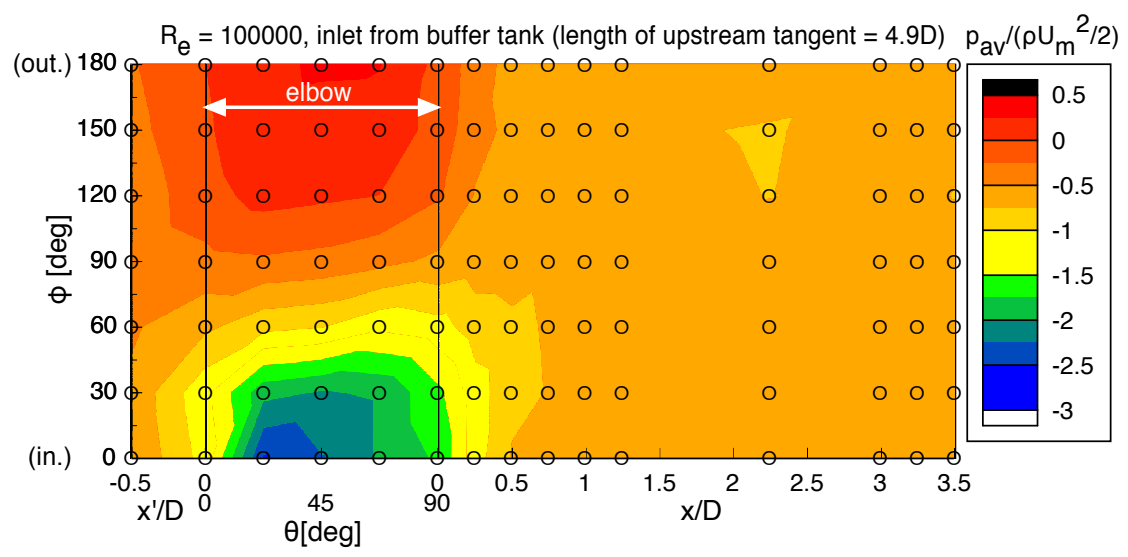

(a) $R_{e}=1.0 \times 10^{5}$

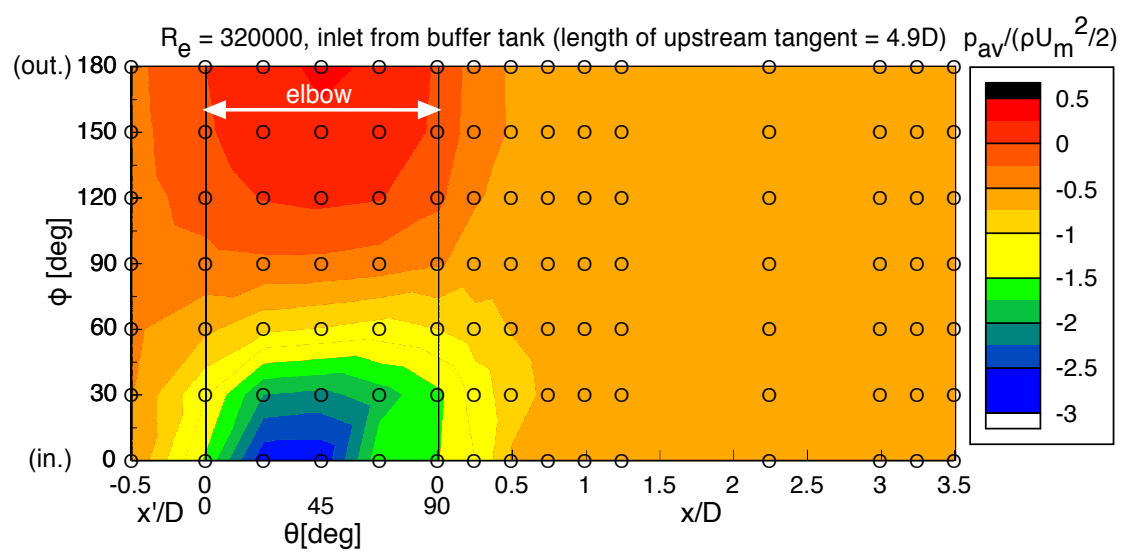

(b) $R_{e}=3.2 \times 10^{5}$

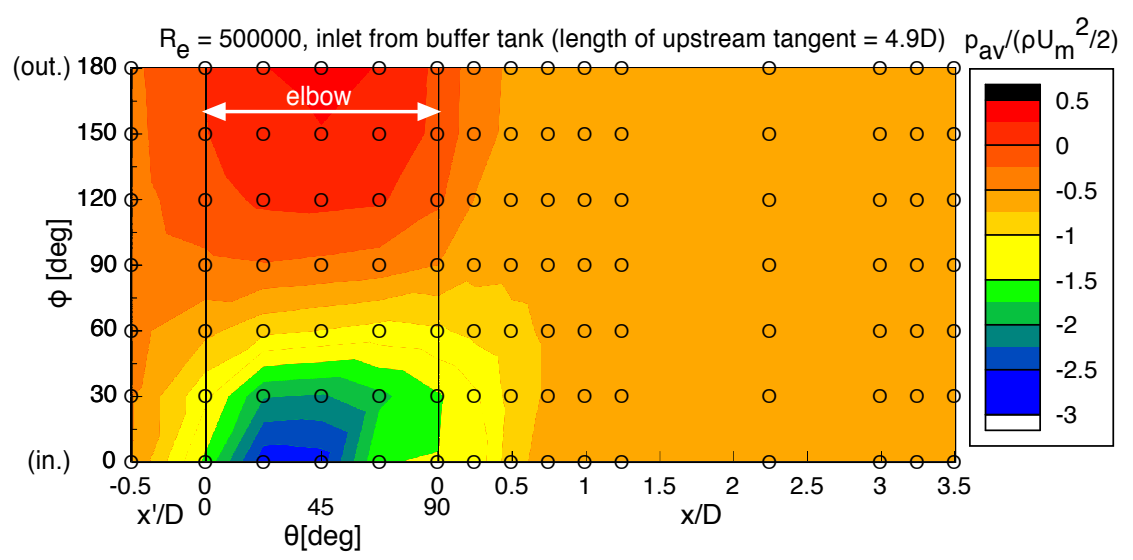

(c) $R_{e}=5.0 \times 10^{5}$

Fig. 3 Distributions of ensemble averaged pressures

at Point B6, as mentioned before. The result at Point B6 was calculated from raw data. Ordinates show nondimensional power spectral density functions using the square of the dynamic pressure, $\left(\rho U_{m}{ }^{2} / 2\right)^{2}$, and the characteristic time scale related with convection over the pipe, $D / U_{m}$. Abscissae show Strouhal numbers, i.e. nondimensional frequencies using the characteristic time scale. Assumption that advection of vortices promotes the pressure fluctuation (Taylor's frozen hypothesis) supports that the frequency divided by the bulk velocity $f / U_{m}$ is proportional to the reciprocal of the vortex size, a wavenumber $k$ in other words. The inner diameter $D$ is proportional to the integral length scale of turbulence. Thus the Strouhal number $S_{t}$ expresses a normalized wavenumber of the vortex fluctuation using the integral length scale. $S_{t}=1$ corresponds to the vortex fluctuation which size is approximated as the inner diameter of the elbow. According to the Kolmogorov, one-dimensional pressure spectrum $E_{p p}$ is proportional to the wavenumber to the power of $-7 / 3$ in an inertial range of turbulence (Tsuji and Ishihara, 2003). Since $E_{p p}$ is a component of the square of the pressure fluctuation with respect to one wavenumber, it can be converted to $p_{P S D}^{\prime}$, which is a component with respect to one frequency. The 


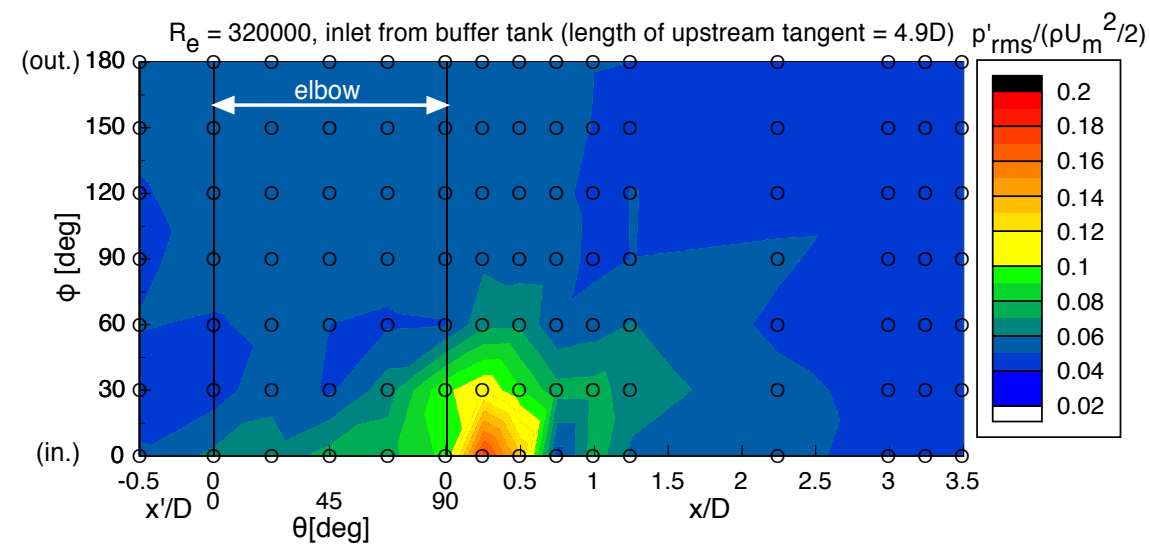

(a) $R_{e}=3.2 \times 10^{5}$

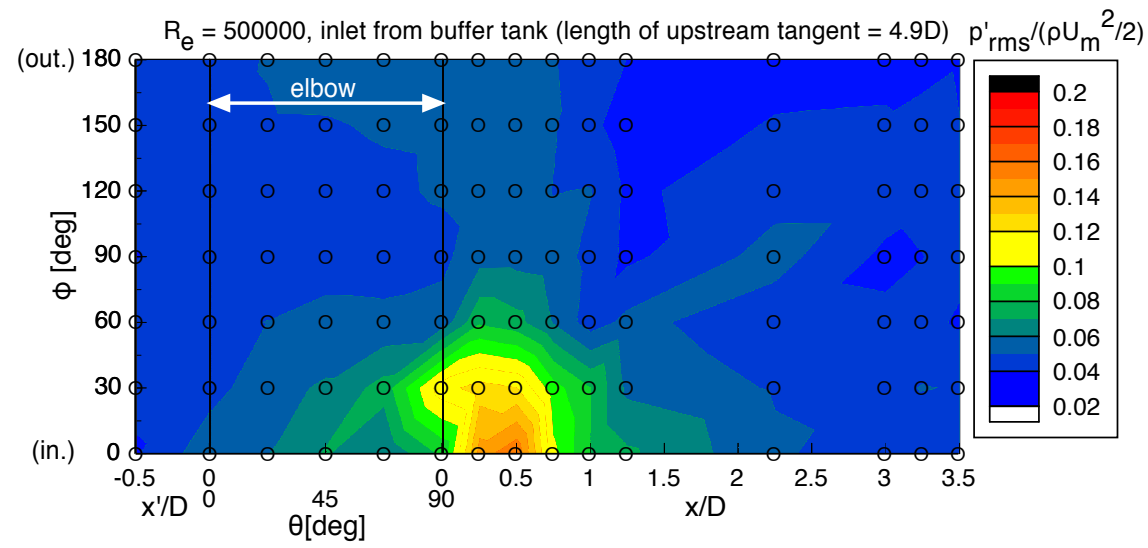

(b) $R_{e}=5.0 \times 10^{5}$

Fig. 4 Distributions of root-mean-square pressure fluctuations

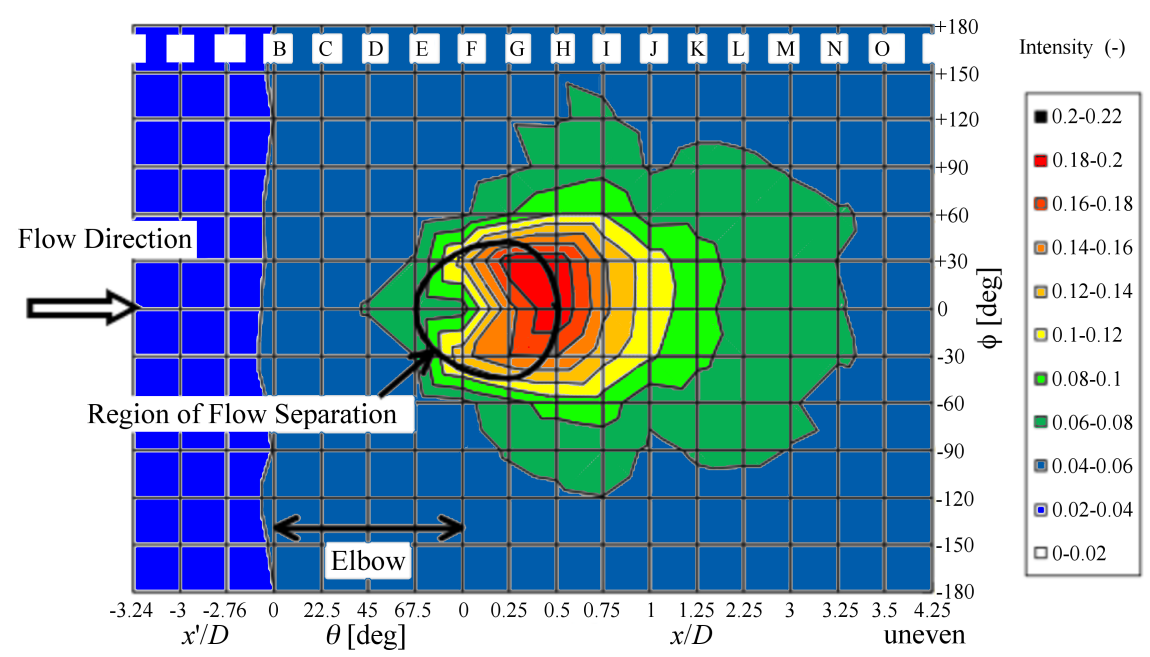

Fig. 5 Distributions of root-mean-square pressure fluctuations obtained by a $1 / 3$-scale model of JSFR hot leg piping. $R_{e}=3.25 \times 10^{6}$. Adapted from Shiraishi et al. (2006). Note that authors have done following modifications: (1) Coloring of contours was done for easier comparison. (2) Values in the ordinate and abscissa were recalculated in order to accommodate the present definition. And (3) Labeling of Cross sections $\mathrm{A}-\mathrm{O}$ were changed to coincide with that of the present study.

description above gives a following relationship:

$$
p_{P S D}^{\prime} \propto \frac{E_{p p}}{U_{m}} \propto \frac{\rho^{2} \epsilon^{4 / 3} k^{-7 / 3}}{U_{m}} \propto \frac{\rho^{2}\left(U_{m}{ }^{3} / D\right)^{4 / 3}\left(S_{t} / D\right)^{-7 / 3}}{U_{m}}=\rho^{2} U_{m}{ }^{3} D S_{t}{ }^{-7 / 3}
$$

where $\epsilon$ is a turbulence dissipation rate, which is proportional to an energy input at large scales $U_{m}{ }^{3} / D$. Dashed lines with the slope of the $-7 / 3$ powers are also drawn in Fig. 6. 

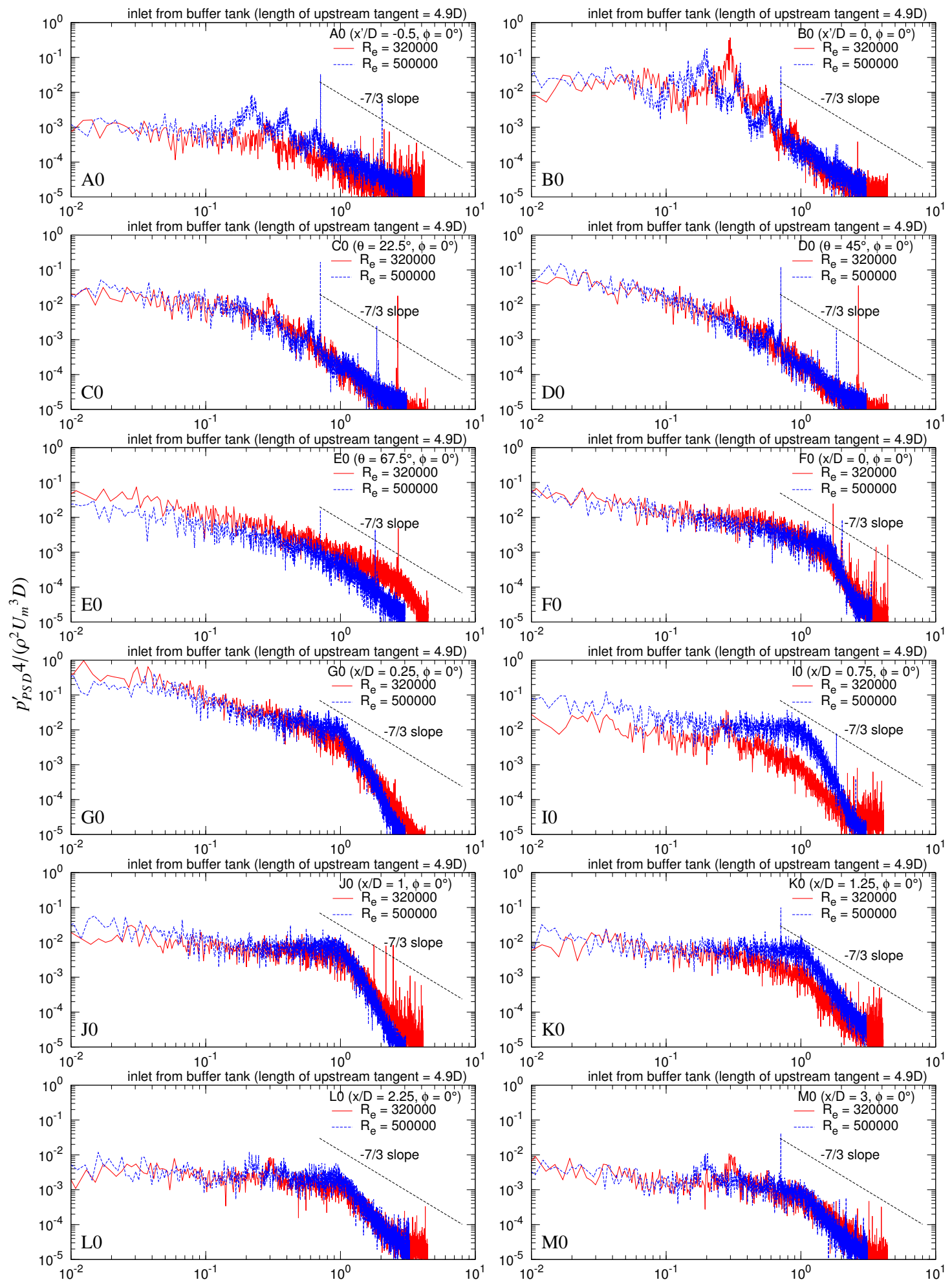

$S_{t}=f D / U_{m}$

(a) inside of the elbow

Fig. 6 Power spectral density functions of pressure fluctuations at various locations

Power spectral density functions for $R_{e}=3.2 \times 10^{5}$ and $5.0 \times 10^{5}$ coincide for almost all the cases in Fig. 6 . It should be noted that our plural measurements occasionally have resulted in different power spectral density functions. The difference was seen in the vertical shift in Fig. 6 or the attenuation rate in higher frequencies. Reasons of this discrepancy are thought as follows: (1) Precision of transducers may not be sufficient for small values in higher frequencies. Repeat accuracy of transducers used is nominally $0.5 \mathrm{kPa}$ at most. This is estimated at $6 \times 10^{-4}$ of a non-dimensional PSD used in 



$S_{t}=f D / U_{m}$

(b) outside of the elbow

Fig. 6 (Continued) Power spectral density functions of pressure fluctuations at various locations

Fig. 6 at $R_{e}=3.2 \times 10^{5}$. And (2) Thicknesses of a seal tape wrapping transducers are inconsistent, causing a variance of the volume in front of the transducer. In the present study, data with high reproductivity have been selected as the correct result in the occurrence of discrepancy.

Figure 6 shows that power spectral density functions in $S_{t}>1$ attenuate in proportion to the $-7 / 3$ powers of the Strouhal number, except Points F0 - L0 $\left(0 \leq x / D \leq 2.25, \phi=0^{\circ}\right)$, which are inside and downstream the elbow. This 
suggests that scales of vortices in locations except Points F0 - L0 so widely spread in extent that measurements include fluctuations in the inertial range of turbulence. By contrast, Points F0 - L0 exhibit sudden attenuation. The axial velocity near these points is decreased because of the adverse pressure gradient, so the dissipation rate of turbulence $\epsilon$ (proportional to the cube of the local velocity) is thought to be small. This leads to the Kolmogorov's dissipation scale $v^{3 / 4} / \epsilon^{1 / 4}$ to be larger than the other locations, resulting in dissipation at smaller wavenumbers (i.e. smaller Strouhal numbers). This may be preferable in LES numerical calculations, since additional refinements of computational grids in downstream regions are not needed so long as grids used can resolve the inertial range of turbulence in the elbow. Considering that large scale vortices shed from the boundary layer thickening at the elbow intrados pass through the region including Points F0 - L0 with diffusion, there is another possibility that these vortices affect PSD distributions.

We have confirmed that all the data shown here do not change their slopes in higher Strouhal numbers $\left(S_{t}>1\right)$ when processed without subtractions. Here one question emerges about the subtraction technique mentioned in Section 3: Why the subtracted data of which power in the inertial regime is also removed still exhibit slopes of the $-7 / 3$ powers? One of the reasons for this is that the reference data at Point B6 $\left(x^{\prime} / D=0, \phi=180^{\circ}\right)$ are relatively smaller than original raw data in the corresponding regime. Another reason, which especially applies to outside points at $R_{e}=5.0 \times 10^{5}$, is that the corresponding regime has scatter in PSD intensities and phases calculated from plural FFT analyses; The final PSD result has been obtained by ensemble averaging over results from five FFT analyses, as mentioned in Section 3. Table 1 shows results of five FFT analyses for $S_{t}=1.1$ obtained at Point I6 $\left(x / D=0.75, \phi=180^{\circ}\right)$ in $R_{e}=5.0 \times 10^{5}$. Subtractions have been done before each analysis. Not shown here, but we confirmed that the raw data at Point I6 has the same PSD magnitude as that at the reference point B6 in higher Strouhal numbers $\left(S_{t}>1\right)$. Table 1 illustrates that every FFT analyses have scatter both in intensities and phases. Assuming that the scattering is caused by random errors, averaging over the results can reduce the errors in PSDs. The PSD with the subtraction $p_{P S D}^{\prime}$ has following relationship according to the cosine theorem:

$$
p_{P S D}^{\prime}(\boldsymbol{x}) \propto \widetilde{p_{\text {raw }}^{\prime}}(\boldsymbol{x})^{2}+\widetilde{p_{\text {raw }}^{\prime}}\left(\boldsymbol{x}_{B 6}\right)^{2}-2 \widetilde{p_{\text {raw }}^{\prime}}(\boldsymbol{x}) \widetilde{p_{\text {raw }}^{\prime}}\left(\boldsymbol{x}_{B 6}\right) \cos \sigma
$$

Here $\boldsymbol{x}$ is a location to be evaluated, $\boldsymbol{x}_{B 6}$ the location of Point B6, $\widetilde{p_{\text {raw }}^{\prime}}$ is amplitude of an oscillation resolved by a FFT analysis without the subtraction, and $\sigma$ the phase measured from Point B6. As is seen in Table 1, the phase $\sigma$ takes wide scatter between $\pm 180^{\circ}$. It is considered that the phase shift between the evaluation and reference points do not have correlation since vortices in the internal range are convected with fluctuation and decrease their size (increase frequencies) by stretching while being convected from the reference to evaluation point. The sufficient average over scattered phases completely reduces the last term of Eq. (2), and the sum of squares of pressure fluctuations at the evaluation and reference points remains. In the present case, both the evaluation and reference points have slopes of the $-7 / 3$ powers in higher Strouhal numbers $\left(S_{t}>1\right)$, resulting in the slope of the $-7 / 3$ powers with the subtraction technique.

Table 1 PSDs and phases $\sigma$ by five FFT analyses at $S_{t}=1.1$. Each analysis has utilized different 4096 samples at Point I6 $\left(x / D=0.75, \phi=180^{\circ}\right)$. The input data are ones from which data at point B6 ( $x^{\prime} / D=0, \phi=$ $180^{\circ}$ ) are subtracted. $R_{e}=5.0 \times 10^{5}$.

\begin{tabular}{c|c|c}
\hline & $p_{P S D^{4}}^{\prime} 4 /\left(\rho^{2} U_{m}{ }^{3} D\right)$ & $\sigma$ \\
\hline 1st & $5.9 \times 10^{-6}$ & $-54^{\circ}$ \\
2nd & $1.5 \times 10^{-4}$ & $-66^{\circ}$ \\
3rd & $9.4 \times 10^{-5}$ & $+14^{\circ}$ \\
4th & $7.7 \times 10^{-5}$ & $-118^{\circ}$ \\
5th & $7.4 \times 10^{-5}$ & $-84^{\circ}$ \\
\hline
\end{tabular}

Power spectral density functions of unsteady pressures at the inside and near the elbow outlet $\left(0 \leq x / D \leq 0.5,0^{\circ} \leq\right.$ $\theta \leq 60^{\circ}$ ) are shown in Fig. 7. The figure includes results for $R_{e}=3.2 \times 10^{5}, 5.0 \times 10^{5}$ and of the $1 / 3$ scale model (Yamano, et al., 2011). Fig. 7 shows a good agreement between $R_{e}=3.2 \times 10^{5}$ and $5.0 \times 10^{5}$. Comparison with results of the $1 / 3$ scale model shows that all the data but at the elbow inside $\left(\phi=0^{\circ}\right)$ have spectral peaks with $S_{t}=0.5$, and all the magnitude with respect to these peaks are the same. The spectral peaks with $S_{t}=0.5$ are excited by vortices shed from circumferential edges of the boundary layer at the elbow inside (Iwamoto, et al., 2012). Values of this phenomenon can be summarized as one when nondimensionalized by the bulk velocity and elbow inner diameter. However the attenuation in case of the $1 / 3$ scale model is gradual and close to the slope of the $-7 / 3$ powers. The ratio of the Kolmogorov's dissipation scale $l_{K}$ to the integral scale of turbulence $l_{I}$ can be evaluated as follows:

$$
\frac{l_{K}}{l_{I}} \propto \frac{v^{3 / 4} \epsilon^{-1 / 4}}{D}=\frac{v^{3 / 4}\left(U_{m}^{3} / D\right)^{-1 / 4}}{D}=v^{3 / 4} U_{m}{ }^{-3 / 4} D^{-3 / 4}=R_{e}{ }^{-3 / 4}
$$



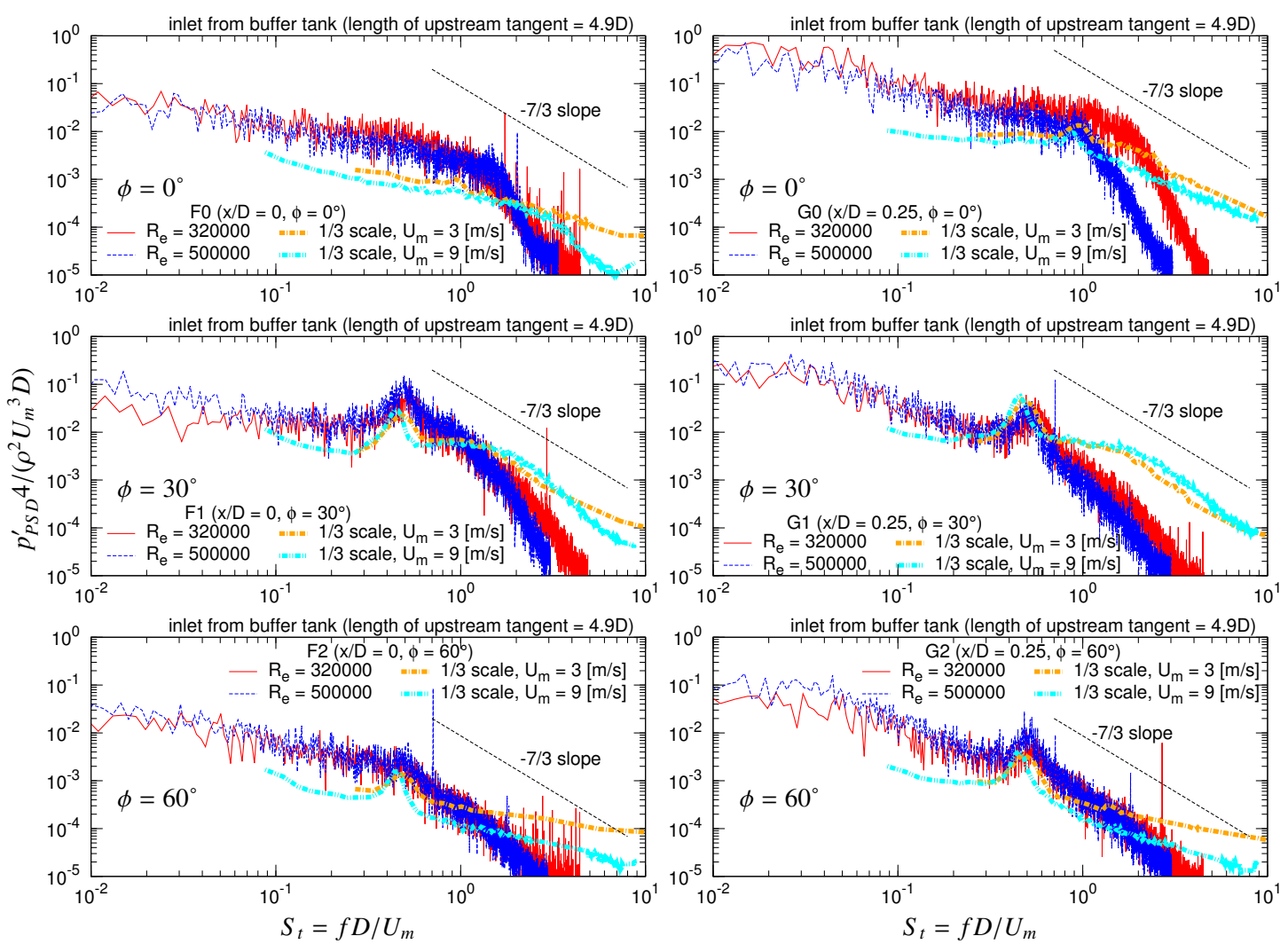

(a) $x / D=0$

(b) $x / D=0.25$
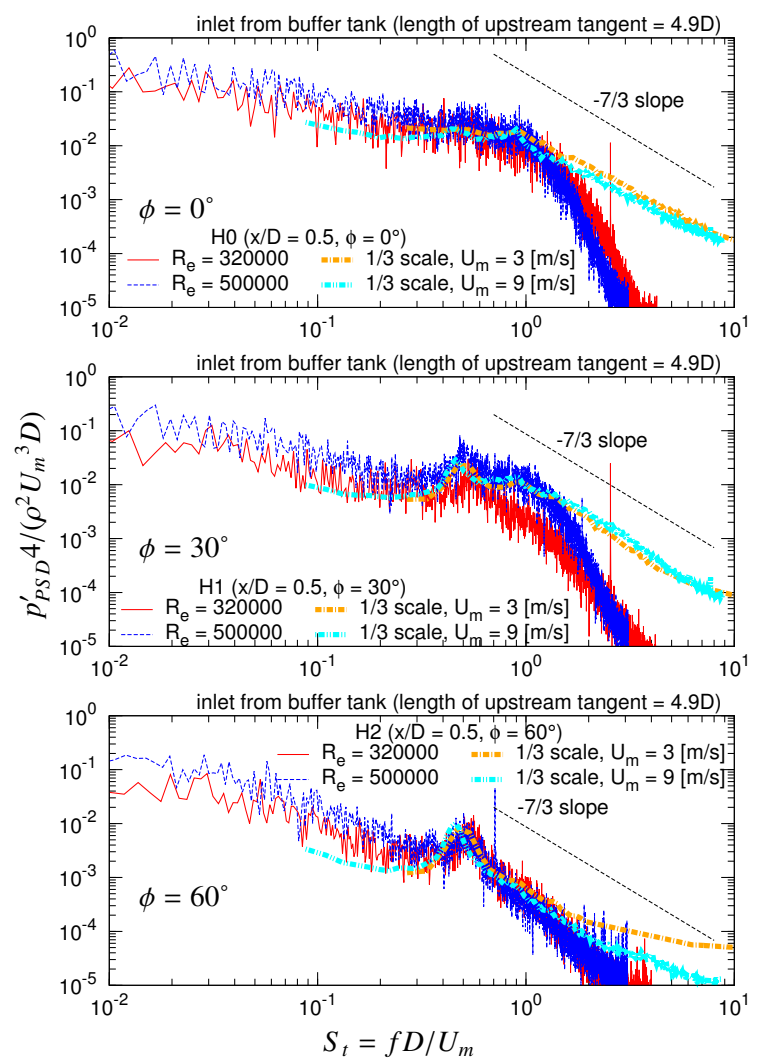

(c) $x / D=0.5$

Fig. 7 Power spectral density functions of unsteady pressures in the region downstream from the elbow. Top, 0 ; center, 30; bottom, 60 degrees circumferentially apart from the elbow inside. Reynolds numbers of $U_{m}=3$ and $9[\mathrm{~m} / \mathrm{s}]$ in $1 / 3$ scale model respectively are $1.06 \times 10^{6}$ and $3.25 \times 10^{6}$. 
Equation (3) means smaller vortices can be exist in higher Reynolds numbers, suggesting that the inertial range of turbulence (exhibiting the slope of the $-7 / 3$ powers) widely exist. Probably this applies to the case of the $1 / 3$ scale model, operating in higher Reynolds numbers $\left(U_{m}=3\right.$ and $9[\mathrm{~m} / \mathrm{s}]$ in Fig. 7 respectively correspond to $1.06 \times 10^{6}$ and $\left.3.25 \times 10^{6}\right)$.

\subsection{Cross correlations of pressure fluctuations}

In order to investigate propagating behavior of pressure fluctuations, cross correlation coefficients have been calculated. Preliminary measurements showed that the characteristic fluctuation with $S_{t}=0.5$, which was caused by vortex shedding, was distinctive at Point G1 $\left(x / D=0.25, \phi=30^{\circ}\right)$, as shown in Fig. 7(b). Therefore all the measurements were made with the simultaneous measurement at Point G1, and the result at Point G1 was used as the basis of the correlation. The cross correlation coefficient evaluated in the present study is expressed as follows:

$$
R_{G 1}(\boldsymbol{x}, \tau)=\frac{\overline{p^{\prime}(\boldsymbol{x}, t) p^{\prime}\left(\boldsymbol{x}_{G 1}, t+\tau\right)}}{\sqrt{\overline{p^{\prime}(\boldsymbol{x})^{2}}} \sqrt{\overline{p^{\prime}\left(\boldsymbol{x}_{G 1}\right)^{2}}}}
$$

Here $p^{\prime}$ is a pressure fluctuation, $\boldsymbol{x}$ a location to be evaluated, $\boldsymbol{x}_{G 1}$ the location of Point G1, $t$ time, and $\tau$ a time delay measured from Point G1. When a large correlation is obtained for some $\tau$, the fluctuation at $\boldsymbol{x}$ is thought to be generated by the fluctuation propagating from/to Point G1, and delays/precedes when $\tau$ is positive/negative. Cross correlation coefficients were actually calculated using FFT: First, values of $p^{\prime}(\boldsymbol{x}, t)$ and $p^{\prime}\left(\boldsymbol{x}_{G 1}, t\right)$ were Fourier transformed. The product of the complex Fourier component of $p^{\prime}(\boldsymbol{x}, t)$ and conjugate one of $p^{\prime}\left(\boldsymbol{x}_{G 1}, t\right)$ was obtained for every frequency, generating a cross spectrum. The cross correlation was finally obtained by the inverse Fourier transformation of the cross spectrum. The input data for the cross correlation consisted of 16384 samples with a sampling frequency of $200 \mathrm{~Hz}$. Therefore the time delay $\tau$ in Eq. (4) ranges between $\pm 16384 / 2 \times 1 / 200= \pm 40.96$ seconds, which is $\pm 10 D / U_{m}$ at least for Reynolds numbers of $3.2 \times 10^{5}$ and $5.0 \times 10^{5}$.

We have extracted largest correlation coefficients $R_{G 1}$ for each measurement. Values of these coefficients and time delays are respectively plotted in Figs. 8 and 9. Lengths of abscissae inside the elbow $\left(0^{\circ} \leq \theta \leq 90^{\circ}\right)$ in both figures are decided by the arc length of the pipe center.

Figure 8 shows fluctuations observed at point G1 start to grow at $\theta=45^{\circ}$ for $R_{e}=3.2 \times 10^{5}$ and $5.0 \times 10^{5}$. This location corresponds to where the adverse pressure gradient emerges at the elbow intrados (see Figs. 3(b) and (c)). It seems that the fluctuations are triggered by the adverse pressure gradient, i.e. the thickening boundary layer.

Dashed lines in the Fig. 9 are drawn assuming that fluctuations are convected at the bulk velocity. Two dashed lines in each figure are vertically separated assuming that the fluctuations have the periodicity with $S_{t}=0.5$. Symbols for $30^{\circ} \leq \phi \leq 120^{\circ}$ surround these lines, suggesting that some of the fluctuations observed at Point G1 are excited by the planar wave convected at the bulk velocity. The points along with the lower line in each figure illustrate the fluctuation in the preceding period, i.e. the fluctuation has periodicity with $S_{t}=0.5$. It has been proved that the fluctuation of $S_{t}=0.5$ can be found by calculating cross correlations, and travels with the bulk velocity as the planar wave. The symbols surrounding the line can be found in the elbow inside $\left(0^{\circ} \leq \theta \leq 90^{\circ}\right)$. This means that the fluctuation of $S_{t}=0.5$ begins to grow at the elbow entrance, not the thickening boundary layer in the aft part of the elbow.

\section{Conclusion}

Wall pressures have been measured for a 90 degree elbow of which the axis curvature coincides with its inner diameter. The conclusion obtained can be listed as follows:

( 1 ) Distributions of ensemble averaged nondimensional pressure for $R_{e}=1.0 \times 10^{5}, 3.2 \times 10^{5}$ and $5.0 \times 10^{5}$ becomes similar, except the region latter part of the elbow at the inside. The difference is considered to appeared depending on whether the flow separation occurs or not.

(2) Distributions of fluctuating normalized pressures for $R_{e}=3.2 \times 10^{5}, 5.0 \times 10^{5}$ and that of the $1 / 3$ scale model done by Shiraishi et al. (2006) coincide within 0.04 of the dynamic pressure.

( 3 ) Power spectral density functions of pressure fluctuations for $R_{e}=3.2 \times 10^{5}, 5.0 \times 10^{5}$ in the present study show that they have the region which obeys the proportionality with respect to $-7 / 3$ powers of the Strouhal number, i.e. the inertial range of turbulence.

( 4 ) Sudden attenuation of pressure fluctuations can be seen in the region downstream and inside of the elbow.

( 5 ) Fluctuations of $S_{t}=0.5$, caused by the vortex shedding from the boundary layer occurring at the elbow inside, have the same levels of power spectral density functions between our experiments and those of the $1 / 3$ scale model. 


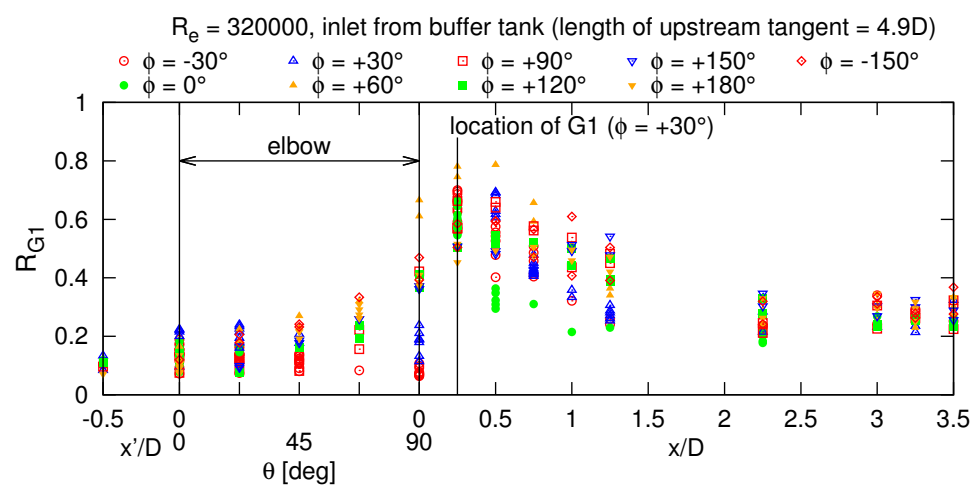

(a) $R_{e}=3.2 \times 10^{5}$

$R_{\mathrm{e}}=500000$, inlet from buffer tank (length of upstream tangent $=4.9 \mathrm{D}$ )

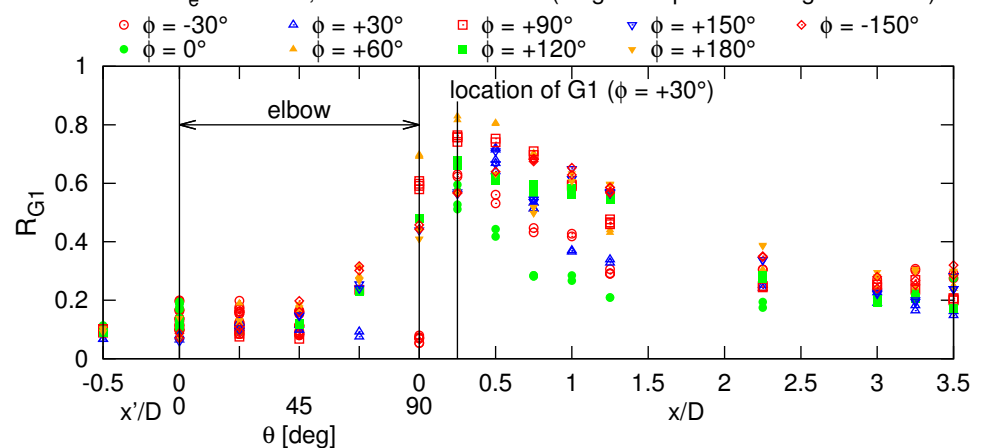

(b) $R_{e}=5.0 \times 10^{5}$

Fig. 8 Large cross correlation coefficients of pressure fluctuations with respect to those at Point $\mathrm{G} 1(x / D=$ $\left.0.25, \phi=30^{\circ}\right)$

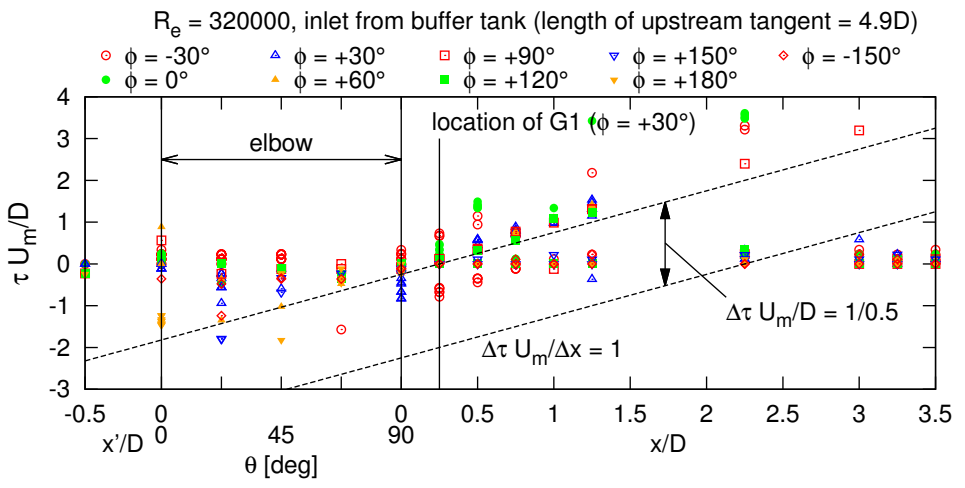

(a) $R_{e}=3.2 \times 10^{5}$

$R_{e}=500000$, inlet from buffer tank (length of upstream tangent $=4.9 \mathrm{D}$ )

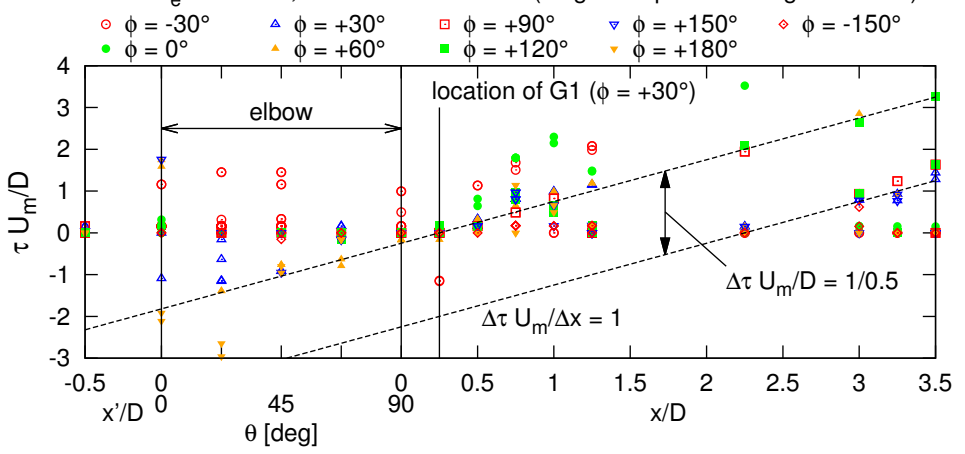

(b) $R_{e}=5.0 \times 10^{5}$

Fig. 9 Time delays $\tau$ measured from Point $\mathrm{G} 1\left(x / D=0.25, \phi=30^{\circ}\right)$ for fluctuations having large cross correlation coefficients 
( 6 ) The discrepancy of power spectral density functions between our measurements and those of the $1 / 3$ scale model is caused by the difference of the extent between the largest vortex length scale and the Kolmogorov's dissipation scale.

( 7 ) The fluctuation having the Strouhal number of 0.5 starts to grow at the inlet of the elbow, and propagates as a planar wave with the bulk velocity.

\section{References}

Crawford, N. M., Cunningham, G. and Spence, S. W. T., An Experimental Investigation into the Pressure Drop for Turbulent Flow in $90^{\circ}$ Elbow Bends, Proceedings of the Institution of Mechanical Engineers, Part E: Journal of Process Mechanical Engineering, Vol. 221, No. 2 (2007), pp. 77-88.

Ebara, S., Aoya, Y., Sato, T., Hashizume, H., Yuki, K., Aizawa, K. and Yamano, H., Pressure Fluctuation Characteristics of Complex Turbulent Flow in a Single Elbow With Small Curvature Radius for a Sodium-Cooled Fast Reactor, Transactions of the ASME, Journal of Fluids Engineering, Vol. 132, No. 11 (2010), 111102.

Hirota, K., Ishitani, Y., Nakamura, T., Shiraishi, T., Sago, H., Yamano, H. and Kotake, S., Flow-Induced Vibration of a Large-Diameter Elbow Piping in High Reynolds Number Range; Random Force Measurement and Vibration Analysis, Proceedings of the 9th International Conference on Flow-Induced Vibration (FIV2008), (2008), Paper No. 264.

Idelchik, I. E., Handbook of Hydraulic Resistance (Second Edition) (1986), pp. 271-275, Hemisphere Publishing.

Ito, H., Pressure Losses in Smooth Pipe Bends, Transactions of the ASME, Series D, Journal of Basic Engineering, Vol. 82, No. 1 (1960), pp. 131-143.

Iwamoto, Y., Kondo, M., Minamiura, H., Tanaka, M. and Yamano, H., Unsteady Flow Characteristics in a 90 Degree Elbow Affected by Developed, Undeveloped and Swirling Inflow Conditions, Journal of Fluid Science and Technology, Vol. 7, No. 3 (2012), pp . 315-328.

Kotake, S., Sakamoto, Y., Mihara, T., Kubo, S., Uto, N., Kamishima, Y., Aoto, K. and Toda, M., Development of Advanced Loop-Type Fast Reactor in Japan, Nuclear Technology, Vol. 170, No. 4 (2010), pp. 133-147.

Miller, D. S., Internal Flow Systems, 2nd Edition (1990), pp. 71-81, BHR Group.

Rütten, F., Schröder, W. and Meinke, M., Large-Eddy Simulation of Low Frequency Oscillations of the Dean Vortices in Turbulent Pipe Bend Flows, Physics of Fluids, Vol. 17 (2005), 035107.

Shiraishi, T., Watakabe, H., Sago, H., Konomura, M., Yamaguchi, A. and Fujii, T., Resistance and Fluctuating Pressures of a Large Elbow in High Reynolds Numbers, Transactions of the ASME, Journal of Fluids Engineering, Vol. 128, No. 5 (2006), pp. 1063-1073.

Tsuji, Y. and Ishihara, T., Similarity Scaling of Pressure Fluctuation in Turbulence, Physical Review E, Vol. 68 (2003), 026309.

Yamano, H., Tanaka, M., Kimura, N., Ohshima, H., Kamide, H. and Watanabe, O., Development of Flow-Induced Vibration Evaluation Methodology for Large-Diameter Piping with Elbow in Japan Sodium-Cooled Fast Reactor, Nuclear Engineering Design, Vol. 241 (2011), pp. 4464-4475. 